\title{
STAR CLUSTERS IN INTERACTING AND COOLING FLOW GALAXIES ${ }^{1}$
}

\author{
Jon A. Holtzman and Alan M. Watson \\ Department of Astronomy, New Mexico State University, Dept 4500, Box 30001, Las Cruces, New Mexico 88003 \\ Electronic mail: holtz@nmsu.edu, awatson@nmsu.edu
}

JEREMY R. MOULD

Mount Stromlo and Siding Springs Observatories, Australian National University, Private Bag, Weston Creek Post Office, ACT 2611, Australia

Electronic mail: jrm@merlin.anu.edu.au

JOHN S. GALLAGHER III

Department of Astronomy, University of Wisconsin-Madison, 475 N. Charter St., Madison, Wisconsin 53706 Electronic mail: jsg@astro.wisc.edu

GILDA E. BALLESTER

Department of Atmospheric, Oceanic, and Space Sciences, University of Michigan, 2455 Hayward, Ann Arbor, Michigan 48109

\section{Christopher J. BURROWS}

Astrophysics Division, Space Science Department, ESA \& Space Telescope Science Institute, 3700 San Martin Drive, Baltimore, Maryland 21218

JOHN T. ClARKE

Department of Atmospheric, Oceanic, and Space Sciences, University of Michigan, 2455 Hayward, Ann Arbor, Michigan 48109

DAVID CRISP AND ROBIN W. EVANS

Jet Propulsion Laboratory, 4800 Oak Grove Drive, Pasadena, California 91109

\section{RICHARD E. GRIFFITHS}

Department of Astronomy, Johns Hopkins University, 3400 N. Charles St., Baltimore, Maryland 21218

\section{J. JEFF HESTER}

Department of Physics and Astronomy, Arizona State University, Tyler Mall, Tempe, Arizona 85287

JOHN G. HOESSEL

Department of Astronomy, University of Wisconsin-Madison, 475 N. Charter St., Madison, Wisconsin 53706

PaUl A. SCOWEN

Department of Physics and Astronomy, Arizona State University, Tyler Mall, Tempe, Arizona 85287

KARL R. STAPELFELDT AND JOHN T. TRAUGER

Jet Propulsion Laboratory, 4800 Oak Grove Drive, Pasadena, California 91109

\section{JAMES A. WESTPHAL}

Division of Geological and Planetary Sciences, California Institute of Technology, Pasadena, California 91125 Received 1996 January 3; revised 1996 May 13

\begin{abstract}
Two interacting galaxies and four central galaxies in cooling flow clusters were observed with the Hubble Space Telescope to look for young compact massive star clusters. The two interacting galaxies, NGC 3597 and NGC 6052, show clear evidence for resolved bright blue clusters that are likely to be comparable in mass to Galactic globulars based on their colors and brightnesses. In NGC 3597, the clusters have physical sizes comparable to Galactic globulars. In NGC 6052, there are a few compact clusters, but most appear more extended. No such objects were seen in three of the four cooling flow galaxies. The central galaxy in Abell 1795 may have several massive blue clusters, but its distance prevents us from knowing how compact they are. (0) 1996 American Astronomical Society.
\end{abstract}

\footnotetext{
'Based on observations with the NASA/ESA Hubble Space Telescope, obtained at the Space Telescope Science Institute, operated by AURA Inc under contract to NASA.
} 
TABLE 1. Observed objects.

\begin{tabular}{|c|c|c|c|c|c|}
\hline Object & $\alpha(2000)$ & $\delta(2000)$ & $l$ & $b$ & Alternate designations \\
\hline NGC 3597 & 111442.1 & -234341.6 & 276.02 & 34.04 & \\
\hline NGC 6052 & 160513.0 & +203232 & 35.31 & 45.47 & Mrk 297 \\
\hline Abell 496 & 043337.8 & -131542.5 & 209.59 & -36.49 & MCG-02-12-039 \\
\hline Abell 1795 & 134852.5 & +263535.0 & 33.81 & 77.19 & CGCG $162-010, \mathrm{MCG}+05-33-005$ \\
\hline Abell 2029 & 151056.0 & +54441.4 & 6.47 & 50.55 & IC1101, CGCG 049-023,UGC 9752 \\
\hline Abell 2597 & 232519.7 & -12727.5 & 65.34 & -64.85 & WPM1G-12.0625, PKS2322-12 \\
\hline
\end{tabular}

\section{INTRODUCTION}

In the past several years, young compact massive star clusters have been discovered in a variety of different locations, including dwarf galaxies (Hunter et al. 1994; O'Connell et al. 1994; Conti \& Vacca 1994), interacting galaxies (Whitmore et al. 1993; Whitmore \& Schweizer 1995; Crabtree \& Smecker-Hane 1994), the central cluster galaxy in the Perseus cluster (NGC 1275, Holtzman et al. 1992), starburst galaxies (Watson et al. 1996; O'Connell et al. 1995), and in ring galaxies (Barth et al. 1995). Somewhat less massive examples of young compact clusters can also be found in the Large Magellanic Cloud. These objects are interesting because they appear to be analogs of young Galactic globular clusters. As such, they may provide clues about conditions in the early universe, when the formation of globulars was common. It is not yet clear, however, whether some unifying physical conditions exist in all of the galaxies in which these star clusters have been seen. Understanding the formation of globulars may also be important for understanding the formation of elliptical and spiral galaxies, because the specific frequency of globulars is higher in ellipticals than in spirals (e.g., Zepf \& Ashman 1993).

One of the earliest detections of young compact massive clusters was in NGC 1275. The origin of the clusters in this galaxy is particularly difficult to determine because of the myriad peculiarities of the host. Holtzman et al. (1992) consider three possibilities: an interaction with another galaxy several hundred million years ago, a current interaction with a gas rich galaxy, and formation in the cooling flow which is centered on the galaxy. They find that a current interaction is unlikely to have triggered the formation of the observed clusters, and suggest that the uniformity of cluster colors is more naturally explained with a past interaction than with steady formation in a cooling flow. However, Richer et al. (1993) argue that formation in a cooling flow is more likely.

The subsequent discovery of young compact massive clusters in several other interacting galaxies has lent support to the idea that interactions can trigger cluster formation. However, it is possible that the formation of clusters may not be directly related to interactions; rather, cluster formation could be a facet of a mode of star formation which occurs in interactions, and possibly in other locations as well. If this is the case, it is of interest to find all of the different locations where young massive clusters are seen and to look for some unifying physical conditions.

To better distinguish between the two leading hypotheses for cluster formation in NGC 1275, and to try to find more young compact massive clusters, we observed several inter- acting and cooling flow galaxies with the Wide Field Planetary Camera 2 on the Hubble Space Telescope. Observations were made of the interacting galaxies NGC 3597 and NGC 6052. NGC 3597 shows evidence for young clusters from the ground (Lutz 1991), although ground-based resolution is not sufficient to determine whether these clusters are as compact as globulars. NGC 6052 has also been observed to have bright blue knots (Hequet et al. 1987). The central galaxies in Abell 1795, Abell 496, Abell 2029, and Abell 2597 were also observed; these clusters are among those with the largest inferred mass inflow rates from x-ray observations (Fabian 1992). These galaxies also sample a range of color properties observed in cooling flow galaxies (McNamara \& O'Connell 1992); Abell 1795 and Abell 2597 are unusually blue in their centers, while the other two galaxies have colors closer to those expected for "normal" ellipticals. Table 1 lists the objects and includes alternate designations for the galaxies; in the remainder of this paper, we will refer to the central galaxies by the Abell number of the cluster.

The key contribution of HST to this problem is that it enables us to put interesting limits on the sizes of star clusters and to measure accurate colors. Massive regions of star formation, e.g., giant $\mathrm{H}$ II regions or super associations, are common, but these are not compact and will probably not evolve into things like globular clusters. A cluster with a size comparable to a globular cluster can only be resolved within a few Mpc from the ground; HST extends this capability roughly an order of magnitude in distance. Even with $H S T$, globular clusters in most of our sample of cooling flow galaxies will be unresolved, so we can only place upper limits on the sizes of star clusters found in these objects. Nevertheless, these upper limits are probably enough to distinguish giant $\mathrm{H}$ II regions from compact clusters. Because size is the key, we focus our discussion on the detection of compact massive star clusters.

Section 2 discusses the observations and describes how we measured brightnesses and sizes of cluster candidates in our objects. Section 3 presents the results for the different galaxies, and Sec. 4 summarizes the implications of our observations.

\section{OBSERVATIONS}

Observations were obtained of NGC 3597, NGC 6052, Abell 1795, and Abell 496 in 1994 February, shortly after the first $H S T$ servicing mission. These observations were made through the F555W and F702W filters. Observations of Abell 2029 and Abell 2597 were made in 1995 June and July 
TABLE 2. Observations.

\begin{tabular}{lcll}
\hline \hline Object & Date & Filter & Exposures (sec) \\
\hline NGC 3597 & 19 Feb 94 & F555W & $100,500,500$ \\
& & F702W & $100,500,500$ \\
NGC 6052 & 24 Feb 94 & F555W & $100,500,500$ \\
& & F702W & $100,500,500$ \\
Abell 496 & 25 Feb 94 & F555W & $180,800,800$ \\
& & F702W & $180,800,800$ \\
Abell 1795 & 24 Feb 94 & F555W & $180,800,800$ \\
& & F702W & $180,800,800$ \\
Abell 2029 & 22 June 95 & F450W & 1200,1300 \\
& & F702W & 1000,1100 \\
Abell 2597 & 5 July 95 & F450W & 1200,1300 \\
& & F702W & 1000,1100 \\
\hline \hline
\end{tabular}

through the F450W and F702W filters. In all cases, multiple exposures were taken through each filter to allow cosmic ray events to be identified. Table 2 lists all of the exposure times. We switched from the F555W filter to the F450W filter because the latter provides a better color baseline, although this comes at the expense of decreased signal-to-noise. In all cases, the galaxies were roughly centered in the Planetary Camera, which has a pixel scale of $0.0455 \mathrm{arcsec} / \mathrm{pixel}$.

The images were processed using the techniques outlined by Holtzman et al. (1995a). The early observations were made when the WFPC2 CCDs were at a temperature of $-76^{\circ} \mathrm{C}$ and suffered from a fairly large population of hot pixels. These were removed with delta dark frames taken within a few days of the observations; in subsequent photometry, however, we were careful to avoid measurements near the sites of known hot pixels, since their dark subtraction is not perfect (Holtzman et al. 1995a). The exposures were combined and averaged, rejecting cosmic ray events in the process.

The combined frames for NGC 3597 and NGC 6052 are shown in Figs. 1 and 2 (Plates 20 and 21). NGC 3597 clearly shows a population of reasonably isolated compact blue objects. NGC 6052 is more confused, with cluster-like objects which are embedded in more distributed light. The cooling flow galaxies are more distant, making similar clusters less conspicuous, but nevertheless, compact luminous blue clusters seem to be genuinely absent in most of these, as discussed below. We defer publication of pictures of the cooling flow galaxies to separate papers on the distribution of gas, dust, and stars in cooling flow galaxies (Pinkney et al. 1996; Pinkney et al., in preparation).

\subsection{Photometry}

To measure brightnesses and colors of potential cluster candidates, we performed aperture photometry on the images; we used aperture photometry because, as discussed below, some of the sources are resolved. We selected objects manually because of problems with remnant hot pixels and cosmic rays; the former were more troublesome for the earlier data sets taken when the WFPC2 CCDs were operating at a warmer temperature, and the latter were more of a problem for the later data sets, in which only two (as opposed to
TABLE 3. Adopted galaxy parameters.

\begin{tabular}{llllc}
\hline \hline Object & $m-M$ & $A_{V}$ & $A_{R}$ & scale (pc/pixel) \\
\hline NGC 3597 & 33.4 & 0.12 & 0.08 & 10.6 \\
NGC 6052 & 34.04 & 0.31 & 0.23 & 14.2 \\
Abell 496 & 35.6 & 0.07 & 0.053 & 29.1 \\
Abell 1795 & 37.03 & 0.01 & 0.008 & 56.2 \\
Abell 2029 & 37.48 & 0.053 & 0.039 & 69.1 \\
Abell 2597 & 37.59 & 0.05 & 0.037 & 72.0 \\
\hline \hline
\end{tabular}

three) exposures were taken in each filter. Manual identification was adequate since we were looking for relatively bright objects and because we will not discuss luminosity functions and completeness. We excluded objects near the nucleii of the galaxies and any obvious neighboring galaxies. We found several interesting compact galaxies, and we will discuss these in a separate paper.

We performed aperture photometry using several different aperture sizes. Background levels were determined from the mean in an annulus of 15 to 20 pixels radius around each object. The quality of the resulting photometry is variable. For isolated objects far from the galaxy centers, photometry near the precision expected from photon statistics is obtained. For objects closer to the galaxy centers, there are additional errors arising from uncertainties in the background determination. There is also some photometric contamination for objects with neighbors.

\subsection{Sizes}

A good measure of the sizes of cluster candidates is needed to determine if the objects are as compact as globular clusters. We experimented with fitting smeared PSF models directly to the data, but this technique proved difficult to implement because of limited resolution and signal-to-noise, as well as the unknown nature of the intrinsic cluster profile. We finally settled on using the difference between one and two pixel aperture measurements (hereafter $m_{1}-m_{2}$ ), similar to the technique used on WFPCI data by Holtzman et al. (1992) and Whitmore \& Schweizer (1995). The observed $m_{1}-m_{2}$ values were compared with values measured on model clusters convolved with model PSFs. To minimize scatter arising from variations in $m_{1}-m_{2}$ because of PSF variations across the field of view, our procedure was to create a model PSF at the position of each object and subtract the $m_{1}-m_{2}$ value of the model PSF from the value measured on the actual object. Consequently, our $m_{1}-m_{2}$ values are measured differentially with respect to a point source at the same location on the frame and with the same estimated pixel centering.

The PSF models include detailed modelling of variations of both aberrations and pupil functions across the WFPC2 field, as well as the effects of observed telescope jitter, midfrequency structure on the HST mirrors, focus variations, and pixel smearing inside the WFPC2 CCDs: we believe they are more accurate than the model PSFs that are typically used for WFPC2 (e.g., PSFs from the TinyTim software). We compare with model PSFs because they can be used to understand PSF variations arising from pixel centering and 


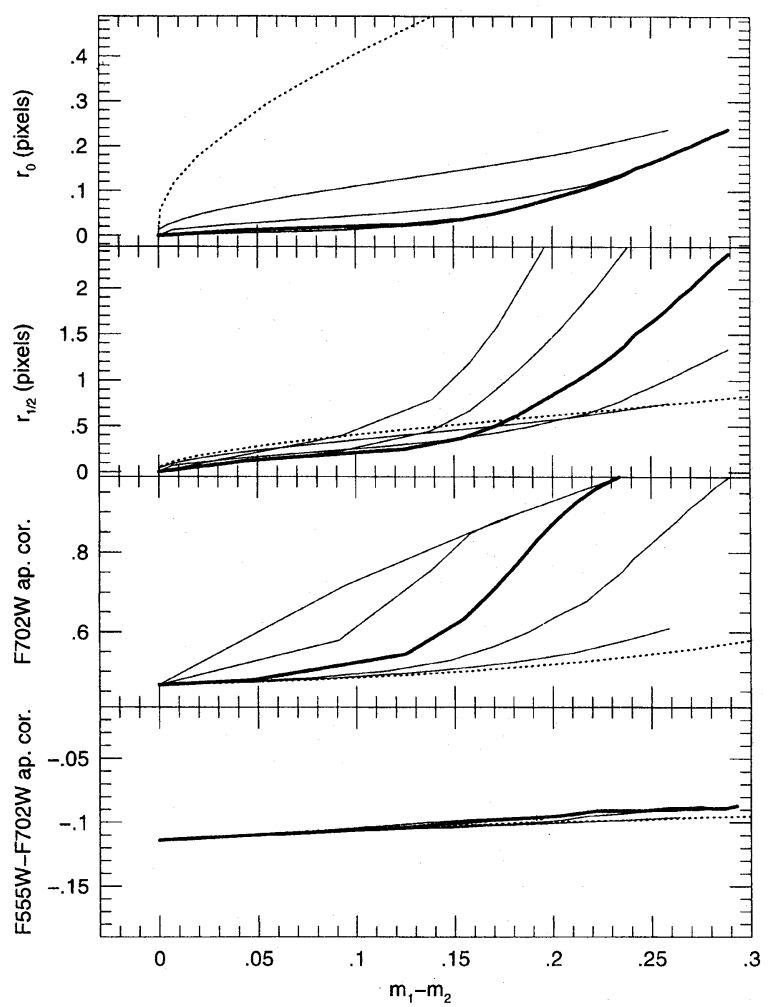

FIG. 3. Sensitivity of derived values of $r_{0}$ (top panel) and $r_{1 / 2}$ (second panel) to $m_{1}-m_{2}$ for several different model cluster profiles. Solid lines are modified Hubble profiles with different ratios of truncation radius to $r_{0}$; ratios of $10,32,100,320$, and 1000 are plotted. The dotted line is for a Gaussian profile. The lower two panels show derived aperture correction for F702W magnitude and for the F555W-F702W color for the same suite of models.

field location and because there are few or no bright stars on our frames. Model PSFs were simulated for each observation using jitter information from each of the individual exposures. However, we found that our size discriminant is relatively insensitive to the exact jitter for these frames, so for our analysis we used just one of the model PSFs. Aside from jitter differences, the PSFs on our combined frames can differ from this model PSF if there are any small pointing changes between the multiple exposure; however, pointing repeatability was good for most of the observations. In cases where frame-to-frame pointing errors are significant, our inferred sizes based on these model PSFs will be slight overestimates. We chose to use the F702W exposures for the measurements of sizes, since they generally have the highest signal-to-noise.

For model cluster profiles, we experimented with a Gaussian profile and also with a modified Hubble law. The latter is given by

$$
I=I_{0}\left(1+\left(\frac{r}{r_{0}}\right)^{2}\right)^{-1},
$$

where $r_{0}$ is the radius at which the surface brightness drops to $1 / 2$ the central value (i.e., the HWHM). The modified Hubble law is a reasonable approximation to a King model and $r_{0}$ corresponds approximately to the core radius of a
King model. Note that we can meaningfully discuss values of $r_{0}$ which are significantly smaller than the resolution of our data. A derived core radius of 0.05 pixels, for example, means that an object has a compactness, judged from one and two pixel aperture photometry, comparable to a King model with a core radius of 0.05 pixels, even though we don't necessarily have information about whether a core of that size actually exists.

An alternate characterization of a cluster size to $r_{0}$ is a half-light radius, $r_{1 / 2}$. However, to measure $r_{1 / 2}$ requires knowledge of the radial extent of an object because one must determine the total integrated brightness. Since the integrated light of a modified Hubble profile diverges at large radii, a truncation radius must be imposed to define a half-light radius. The half-light radius of a Gaussian is well defined and and equal to the HWHM.

From the data, however, the information that we have to measure the total brightness or to derive the shape of the light distribution at large radii is limited because of low $\mathrm{S} / \mathrm{N}$ in the wings of objects and contamination by the background galaxy light. Since we lack information on the shape of the profile for most objects, we consider how well we can determine $r_{0}$ or $r_{1 / 2}$ from $m_{1}-m_{2}$ independently of the assumed radial profile. The top two panels of Fig. 3 show values of $r_{0}$ and $r_{1 / 2}$ as a function of $m_{1}-m_{2}$ for a variety of modified Hubble profiles with truncation radii ranging from $10 r_{0}$ to $1000 r_{0}$ (solid lines), as well as for a Gaussian model (dotted line). Some of the modified Hubble profile curves cross in the second panel because some of the models with small $r_{0}$ have truncation radii smaller than two pixels. For objects measured in NGC 3597, typical values of $m_{1}-m_{2}$ are $0.15-$ 0.2 . For such objects, an accurate $r_{0}$ can be derived fairly independently of truncation radius if the profile is a modified Hubble profile. The derived $r_{1 / 2}$, however, can vary by factors of a few. This is not surprising since the $m_{1}-m_{2}$ measurement probes the inner parts of the cluster profile. If the $\mathrm{S} / \mathrm{N}$ in the outer regions is poor, it is difficult to constrain $r_{1 / 2}$, but easier to constrain $r_{0}$, at least if the profile is similar to a modified Hubble law.

If a Gaussian model is used for a cluster that has an intrinsic modified Hubble profile, reasonable half-light radii will be derived only if the cluster is marginally resolved. For well-resolved clusters, the half-light radii may be significantly underestimated unless the profile has a small truncation radius. This is of interest because several previous analyses of other young clusters (e.g., Whitmore \& Schweizer 1995) use Gaussian profiles to derive sizes.

In subsequent analysis, we consider a modified Hubble profile with a truncation radius of $100 r_{0}$ (bold line in Fig. 3) as an intermediate case between profiles with essentially no wings and profiles with very extended wings, and keep in mind the uncertainties on the derived values of $r_{0}$ and half light radius. This profile also provides a rough match to typical Galactic globulars. We return to the issue of the true profiles briefly when discussing the clusters in NGC 3597 because a few of these objects are isolated and bright enough so that reasonable profiles can be measured, and for these we find that the wings are extended and are much better matched by modified Hubble profiles than by Gaussians. 


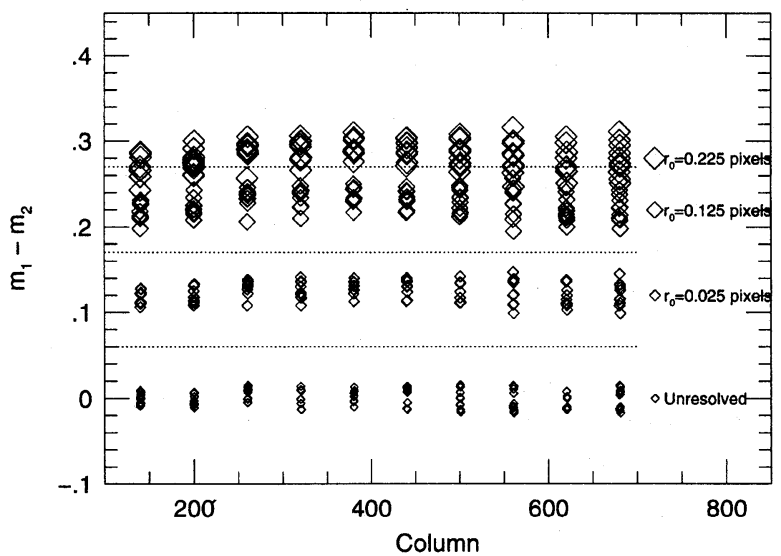

FIG. 4. Measurements of $m_{1}-m_{2}$ for simulated stars. The data are simulated PSFs at a variety of field locations and pixel centerings, and the aperture differences are plotted against the column location. The different sized points refer to models of point sources (smallest points), and models smeared with a modified Hubble profile with $r_{0}=0.025,0.125,0.225$ pixels Dotted lines indicate the regions adopted for different point sizes in Fig. 6.

To demonstrate the sensitivity of $m_{1}-m_{2}$ to cluster size, Fig. 4 shows the $m_{1}-m_{2}$ values for a set of models for the F702W filter, where the models are made from a sample of 100 locations spread across the field of the PC and with a range of different pixel centerings. The different point sizes show results for a point source (smallest points) and for PSFs which have been convolved (before pixel binning) with a modified Hubble law; the aperture differences are plotted against the column location of the PSF for $r_{0}=0.000,0.025$, 0.125 , and 0.225 pixels. The spread seen at each value of $r_{0}$ represents the scatter arising from aperture photometry sensitivities to pixel centering and from centroiding errors, since the model PSFs are constructed at measured pixel centerings. The separation of the different point sizes shows the degree to which angular sizes can be determined from the aperture difference. Objects with $r_{0}$ as small as 0.02 pixels can be resolved; this is not as extraordinary as it sounds if one realizes that such a profile has $\sim 20 \%$ of the light outside of one pixel. Again, we emphasize that we do not claim to detect cores of this size; rather the compactness as measured within the inner two pixels is comparable to a modified Hubble profile with this core radius. Histograms of the aperture differences are shown in the top left panel of Fig. 5; the histograms represent the results for a point source and for the three different core radii shown in Fig. 5. The degree to which the histograms are separated from each other indicates the ability of this technique to determine sizes.

Clearly, however, noise in the measurement of aperture differences implies noise in the inferred cluster sizes. We simulated PSFs with various degrees of noise and histograms of the observed aperture differences are shown in the remaining panels of Fig. 5. As the objects get fainter, there is an increasing amount of mixture of the different PSFs. The panels in Fig. 5 refer to $V$ magnitudes of approximately 22, 23, 24,25 , and 26 (the upper left is for noiseless models), using the exposure times for NGC 3597 and NGC 6052. For the cooling flow galaxies, the exposures were longer, so the cor-

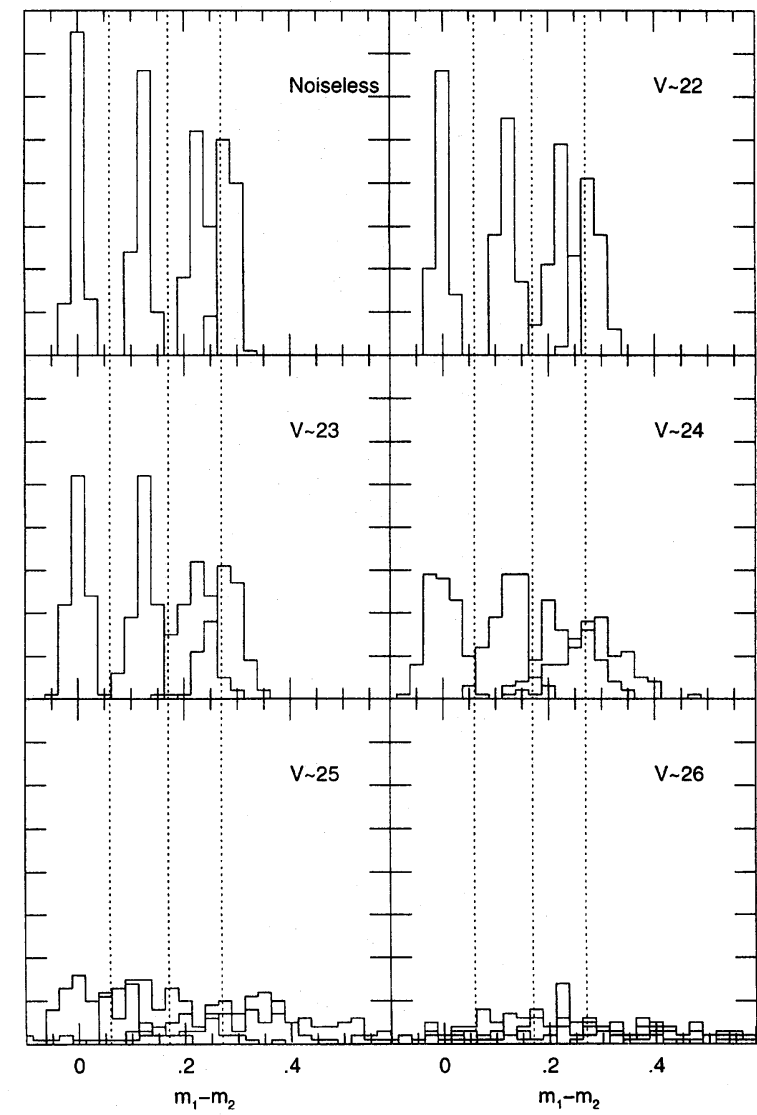

FIG. 5. Histograms of $m_{1}-m_{2}$ for the same simulated stars as in Fig. 4. The upper left panel is noiseless, but other panels include simulated noise appropriate for $V=22,23,24,25,26$ in the NGC 3597 and NGC 6052 exposures; for the other galaxies, the corresponding magnitudes are roughly 0.5 mag fainter since the exposures are longer. Dotted lines indicate the regions adopted for different point sizes in Fig. 6.

responding magnitudes are fainter (by roughly $0.5 \mathrm{mag}$ for Abell 496 and Abell 1795, and 0.7 mag for Abell 2029 and Abell 2597). The correspondence between these simulations and real measurements is only approximate because although the models were computed using good estimates of the gain, readout noise and a typical background level, they do not include the effects of varying background, errors in the background determination, or crowding. One sees that objects can be fairly easily resolved down to $V \sim 24$ for $r_{0} \geq 0.025$, and to fainter magnitudes for larger $r_{0}$.

\subsection{Cluster Magnitudes}

Magnitudes were determined from small aperture measurements by applying an aperture correction and then using the photometric calibration presented by Holtzman et al. (1995b). The size of the aperture correction depends on whether the object is resolved, so the following technique was used.

From the model PSFs, aperture corrections from a 2 pixel radius to a 0.5 arcsec radius (which is used for the photometric calibration) were measured. Aperture corrections of 0.37 
and 0.485 mag for F555W and F702W were adopted for unresolved sources. These values are close (within $2 \%$ ) to the values derived from real observations of stars (e.g., Holtzman et al. 1995a) and also close to values measured on I one object in the NGC 3597 image which we believe is a star. An additional correction based on the measured $m_{1}-m_{2}$ value for each object was then applied to account for the radial extent of the source. This correction was derived from a quadratic fit to the aperture measurements of the model clusters; the correction gives larger aperture corrections for more resolved objects.

For resolved objects, the derived aperture correction depends on the nature of the profile of the object at large radii. As mentioned above, the data provide limited information about this. Consequently our derived integrated brightnesses have some uncertainty. The third panel (from top) of Fig. 3 plots the aperture corrections for the same variety of cluster profiles discussed earlier and shown in the top panels. At the same $r_{0}$, the aperture correction based on $m_{1}-m_{2}$ can vary by several tenths of a magnitude depending on the radial profile and extent of the cluster; this gives an estimate of possible uncertainties on our total magnitudes.

However, since the aperture corrections are similar for each of the two colors for any model, the derived integrated colors are much better determined. This is shown in the bottom panel of Fig. 3, which plots the derived aperture correction for the color for a variety of cluster profiles. This shows essentially no dependence on the assumed profile, and in fact, the derived color is quite insensitive even to whether the object is resolved or not. The mean color correction of $\sim 0.1$ mag arises from the difference in the PSF between the two colors, and errors in the derived color correction should be less than a few percent.

After aperture corrections were applied, the F555W and F702W measurements were transformed to $V$ and $R$ using the synthetic calibration presented by Holtzman et al. (1995b). Uncertainties in the calibration are expected to be less than a few percent; however, systematic errors in the zeropoints may be closer to five percent because of a possible problem in the application of the zeropoints to long exposures (see Note Added in Proof, Holtzman et al. 1995b), and because most of these data were taken at $-76^{\circ} \mathrm{C}$, when possible charge transfer efficiency problems in WFPC 2 were larger than they are at the current operating temperature (Holtzman et al. 1995a). No correction was made for possible CTE effects in these frames because we believe that the galaxy background light provides sufficient background to minimize CTE effects. The transformation to $V$ and $R$ magnitudes was done to facilitate comparison with observations of other clusters and with composite stellar models. These transformations may introduce some additional systematic errors, especially for the F450W observations which require a large color term.

Random error estimates were derived from photon statistics. Systematic errors may result from errors in background determination, aperture correction errors from PSF variations across the field and pixel centering, and possible errors in the calibration zeropoints. Additional systematic errors are present for resolved objects as discussed above. However, we note that all of these systematic effects (except zeropoints) are expected to be independent of stellar color to first order, if they are present at all. Hence, our derived colors should be accurate to within a few percent although each individual magnitude may have a larger systematic uncertainty.

\section{DISCUSSION}

\subsection{Globular Cluster Properties}

Before we present our observational results, we briefly review the properties of globular clusters to put our observations into context. Galactic globulars have a distribution of absolute magnitudes which is roughly Gaussian with a mean of $M_{V} \sim-7.3$ and $\sigma \sim 1.2 \mathrm{mag}$ (Harris \& Racine 1979). Colors are consistent with those expected for an old population; there is some variation with metallicity, leading to observed $V-R$ colors of $0.4-0.55$ (Reed et al. 1988). Estimated masses range between $10^{4}$ and $4 \times 10^{6} M_{\odot}$ (Pryor \& Meylan 1993). Physical sizes vary, but typical core radii are $0.5-5 \mathrm{pc}$ (Djorgovski 1993) and half-light radii are of order 5-10 pc (van den Bergh et al. 1991).

The number of clusters varies significantly from galaxy to galaxy; the number roughly scales with the luminosity of the parent galaxy. In spirals, however, the specific frequency (number of globulars per galaxy luminosity) is lower than in ellipticals, while in central cluster galaxies the fraction can be much larger. The shape of the globular cluster luminosity function is similar in different objects, so a large population of clusters can give a significant number of bright clusters $\left(M_{V}<-10\right)$; M87 has clusters at least as bright as $M_{V}=-11.5$ (Strom et al. 1981; Couture et al. 1990), using a distance of $16 \mathrm{Mpc}$.

If we were to observe globular clusters throughout their lifetime, we would see an evolution of their brightnesses and colors as their stellar populations evolved; generally, clusters get fainter and redder as they age, though there are some periods when the evolution is more complicated, e.g., during the phase when red supergiants dominate the total light. Charlot \& Bruzual (1991) have modelled the evolution of a single age (burst) population with a Salpeter initial mass function and solar metallicity; plausibly, these will approximate the evolution of a present day young globular cluster. The Charlot and Bruzual results for the $V$ magnitude and $V-R$ color evolution have been presented by Holtzman et al. (1992).

One problem with identifying objects as young globular clusters is the difficulty of estimating the probability of survival of an object as a distinct entity over a Hubble time. Young clusters might be destroyed in a variety of ways such as tidal disruption or internal mass loss. The likelihood of tidal disruption would depend on the location and the orbits of the clusters. Since disruption mechanisms or mass loss may have affected the Galactic globular cluster system, it is not clear how the properties of the young Galactic cluster system resemble the current day properties. In the following discussion, we will refer to objects as possible young globulars if their current properties match those of old Galactic 


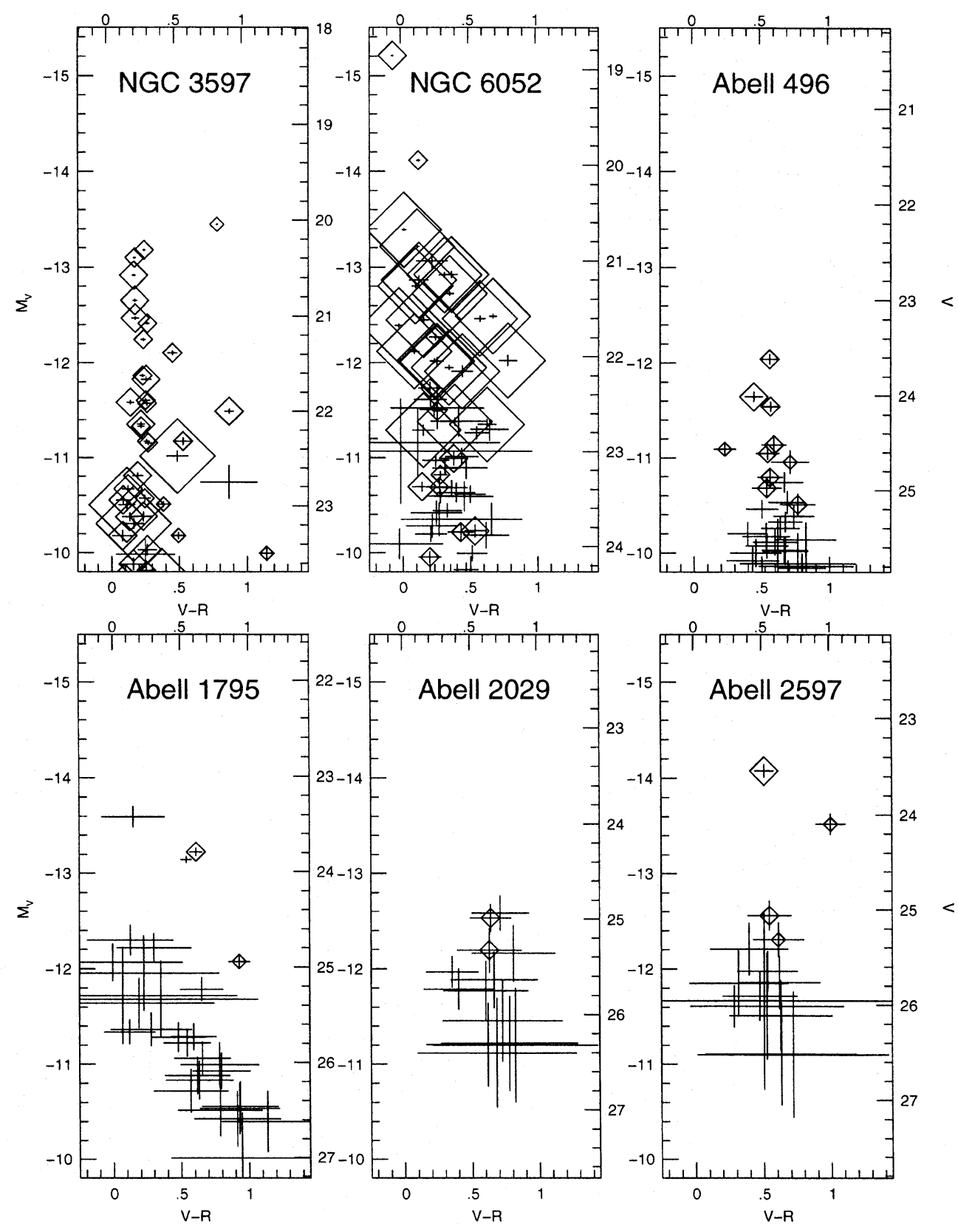

FIG. 6. Color-magnitude diagrams for the objects found in each galaxy. Top and right axes give apparent quantities, and bottom and left give absolute magnitudes using distances and reddenings from Table 3. Point sizes convey size information as described in the text.

globulars, keeping in mind that evolutionary processes may be important.

\subsection{New Observational Results}

The photometry for objects found in each of the galaxies is presented in Fig. 6, which plots $V$ magnitudes of the objects against their $V-R$ color along with our error estimates. In addition, we have coded the inferred angular sizes of the objects into point sizes such that more resolved objects have larger symbols. Objects with large estimated errors have no useful size information, so only error bars are plotted for these. The dotted lines in Figs. 4 and 5 show the bins used for the different point sizes. The smallest points have inferred $r_{0} \leqslant 0.025$ pixels, the next larger points have $0 \leqq r_{0} \leqq 0.1$ pixels, the next size $0.025 \leqslant r_{0} \leqslant 0.25$ pixels, and the largest points have $r_{0} \gtrsim 0.125$ pixels. Note that the ranges for the point sizes overlap because of the intrinsic scatter in $m_{1}-m_{2}$ discussed in Sec. 2.2. These inferred limits are applicable for objects with $V \sim 23$; the constraints on size become weaker as the objects get fainter. The photometry of 
the objects is presented, along with the $m_{1}-m_{2}$ values, in Tables 4-9. These tables also give positions of the measured objects relative to the center of each galaxy.

In Fig. 6, we present both observed (upper and right axes) and absolute (lower and left axes) magnitudes and colors. To convert to absolute magnitudes, we have used the distances and extinctions given in Table 3. Distances were determined using a Hubble constant of $75 \mathrm{~km} / \mathrm{sec} / \mathrm{Mpc}$, and reddenings were estimated from Burstein \& Heiles (1984). No corrections have been applied for possible internal reddening within the galaxies or clusters. Table 3 also shows the physical size of each pixel at the adopted galaxy distance.

None of the objects are at low galactic latitudes (see Table 1), so we do not expect substantial foreground star contamination in the field of view of the PC. We have computed the expected number of Galactic foreground stars based on the Bahcall \& Soneira (1981) model and this confirms our expectation; the predictions average about three stars per field, but most of these are expected to be quite red and faint, with $V-R>0.8$ and $V>25$. Essentially no blue objects are expected, although the model does not include a white dwarf component.

\subsection{NGC 3597}

NGC 3597 clearly shows a population of bright blue objects, which were already known from ground-based observations (Lutz 1991). Typical colors are $V-R \sim 0.25$, and the colors of all of the objects are remarkably uniform, suggesting that there is little internal extinction within NGC 3597 and also that all of the clusters have the same age. The spatial distribution of the clusters appears to be roughly symmetrical around the galaxy nucleus.

Our measured magnitudes and colors are similar to those measured by Lutz (1991) but not identical. Fig. 7 presents a comparison of our magnitudes with those of Lutz, showing the differences in derived $(V-R), V$, and $R$ magnitudes; objects identified as stars (as opposed to clusters) by Lutz are shown with asterisks. Objects with ID numbers less than 10 are found in the Wide Field Camera sections of our images, and were measured directly with 0.5 arcsec apertures. The magnitudes of the other objects were measured as described in Sec. 2. Generally, our derived magnitudes are fainter by a 0.1-0.3 mag for objects which we resolved; agreement is better for the unresolved objects. Our colors, however, agree fairly well. The source of the discrepancy in brightnesses is unclear, although it is likely that it is a result of differences in the aperture corrections. We described our aperture corrections in Sec. 2.3; we do not know how Lutz derived his. In either case, the aperture corrections are uncertain. This interpretation is consistent with the fact that our measured colors agree with those of Lutz better than the individual magnitudes do. However, some of the disagreements certainly arise from errors in the interpretation of the groundbased data: for example, object 19 is actually two objects separated by about 0.5 arcsec and mistaken by Lutz for a single object.

Comparison with the Charlot and Bruzual models suggest ages of less than 500 million years based on the observed color. During the early evolution, the age-color relation for broad-band colors is multivalued, so it is not possible to determine the exact age; colors of $V-R \sim 0.25$ are expected at ages near 10 and 200 million years. However, clusters spend significantly more time near this color at an age of several hundred million years, so this is the more likely age. Additional support for the older age comes from the uniformity of the cluster colors: at the younger age, all clusters would have had to form within a period of about a million years to have the same colors, since color changes rapidly during the early evolution of a cluster. If the clusters have ages of $\sim 200 \mathrm{Myr}$, then the inferred mass is about $1.6 \times 10^{6} M_{\odot}$ for clusters with $M_{v}=-12$. In this case, these objects would fade 3-4 mag by the time they reached an age of 10-15 Gyr. For this age the observed cluster system in NGC 3597 would be comparable to the current Galactic globular clusters, with the brightest clusters in each system being roughly the same mass. If we adopt the less likely younger age, then the NGC 3597 cluster masses would be smaller by about an order of magnitude (which would still leave substantial overlap with the Galactic globulars) and the amount of fading would be several magnitudes larger.

The point sizes in Fig. 6 show that most of the objects are marginally resolved, with inferred core radii (assuming a modified Hubble profile) of less than 0.2 pixels, or 2 pc. To emphasize our ability to resolve these objects and to more accurately constrain sizes, Fig. 8 presents profiles of the four brightest objects, which are relatively isolated and have sufficient $\mathrm{S} / \mathrm{N}$ to allow the measurement of profiles to moderately large radii. The left panel for each object shows the actual data after sky subtraction, and the line is an azimuthal average of the profile derived from aperture photometry. The second panel from the left shows the profile of a model PSF at the same field location and pixel centering. The third panel shows a ratio of the two azimuthally averaged profiles. The model PSF matches the profile of the brightest object quite accurately, but it is clear that the other objects have fainter cores and brighter wings than the model PSF. The rightmost panel shows the same ratio, but this time for a model cluster with $r_{0}=0.05$ pixels $(\sim 0.5 \mathrm{pc})$ after convolution with the model PSF. These data strongly suggest that these objects have core radii of about 0.05 pixels and their profiles match a Hubble profile out to about 3 pixels. A similar comparison with models that have $r_{0}=0.1$ pixels rules out such profiles. At larger radii, the observed profiles differ from the models, suggesting that the models deviate from a modified Hubble profile and have light which extends past the truncation radius of $100 r_{0}=5$ pixels used in the model. We note that in the outer regions the modified Hubble profile deviates significantly from King models, so perhaps this is related to the apparent disagreement with the model. Some of the differences between the data and the models may arise from errors in sky determination, but these errors cannot explain all of the observed differences. The profiles are clearly much more extended than a Gaussian profile.

The derived values of $r_{0}$ are similar to those of Galactic globulars. Hence, our observations confirm the suggestion of Lutz (1991) that these objects are compact enough to be young globular clusters. Of course, for these clusters to eventually become old globular clusters would require that their 
TABLE 4. NGC 3597 objects.

\begin{tabular}{|c|c|c|c|c|c|c|c|}
\hline ID & $\Delta \alpha$ & $\Delta \delta$ & V & $\sigma_{V}$ & $R$ & $\sigma_{R}$ & $m_{1}-m_{2}$ \\
\hline 1 & 11.9 & 6.8 & 20.04 & 0.00 & 19.23 & 0.00 & -0.02 \\
\hline 2 & -8.9 & -9.8 & 20.31 & 0.01 & 20.03 & 0.01 & 0.16 \\
\hline 3 & -11.2 & 7.6 & 20.39 & 0.01 & 20.18 & 0.01 & 0.17 \\
\hline 4 & -3.6 & -17.0 & 20.58 & 0.01 & 20.37 & 0.01 & 0.18 \\
\hline 5 & 11.2 & -0.7 & 21.08 & 0.01 & 20.77 & 0.01 & 0.09 \\
\hline 6 & -10.3 & 0.3 & 20.84 & 0.01 & 20.63 & 0.01 & 0.21 \\
\hline 7 & 8.3 & 4.7 & 21.39 & 0.02 & 20.90 & 0.03 & 0.12 \\
\hline 8 & 3.2 & -5.9 & 21.24 & 0.01 & 20.97 & 0.01 & 0.16 \\
\hline 9 & -4.7 & 15.0 & 21.02 & 0.01 & 20.81 & 0.02 & 0.20 \\
\hline 10 & 6.2 & -6.8 & 21.62 & 0.01 & 21.36 & 0.01 & 0.11 \\
\hline 11 & -7.0 & 1.6 & 21.92 & 0.02 & 21.61 & 0.03 & 0.12 \\
\hline 12 & -7.4 & -2.2 & 21.88 & 0.02 & 21.59 & 0.03 & 0.16 \\
\hline 13 & -3.7 & 4.4 & 21.66 & 0.03 & 21.37 & 0.03 & 0.22 \\
\hline 14 & 16.9 & 11.5 & 22.32 & 0.02 & 22.02 & 0.01 & 0.09 \\
\hline 15 & 6.0 & -15.0 & 22.16 & 0.02 & 21.90 & 0.01 & 0.14 \\
\hline 16 & -5.7 & -1.7 & 22.32 & 0.04 & 21.75 & 0.04 & 0.12 \\
\hline 17 & -12.1 & -8.3 & 21.91 & 0.02 & 21.73 & 0.02 & 0.20 \\
\hline 18 & -12.6 & 2.0 & 22.14 & 0.02 & 21.88 & 0.02 & 0.18 \\
\hline 19 & -3.2 & 6.9 & 22.33 & 0.02 & 22.03 & 0.02 & 0.14 \\
\hline 20 & -5.0 & -5.2 & 22.00 & 0.03 & 21.09 & 0.01 & 0.23 \\
\hline 21 & 8.1 & 3.8 & 22.75 & 0.18 & 21.85 & 0.11 & $\ldots$ \\
\hline 22 & 7.3 & -6.1 & 22.98 & 0.03 & 22.56 & 0.02 & 0.01 \\
\hline 23 & -6.1 & -0.6 & 22.48 & 0.06 & 21.95 & 0.05 & 0.47 \\
\hline 24 & -2.9 & 12.2 & 22.68 & 0.03 & 22.45 & 0.03 & 0.27 \\
\hline 25 & -5.3 & -7.1 & 23.31 & 0.03 & 22.78 & 0.03 & 0.06 \\
\hline 26 & 1.5 & -12.1 & 22.82 & 0.03 & 22.66 & 0.03 & 0.23 \\
\hline 27 & -3.3 & 6.6 & 22.92 & 0.03 & 22.64 & 0.03 & 0.17 \\
\hline 28 & -10.0 & -0.5 & 23.50 & 0.15 & 23.22 & 0.16 & $\cdots$ \\
\hline 29 & 6.1 & 7.7 & 23.50 & 0.05 & 22.31 & 0.02 & 0.01 \\
\hline 30 & 0.3 & 15.2 & 22.94 & 0.04 & 22.82 & 0.04 & 0.26 \\
\hline 31 & 11.9 & -3.4 & 22.99 & 0.05 & 22.84 & 0.04 & 0.34 \\
\hline 32 & -0.2 & -14.7 & 23.11 & 0.04 & 22.94 & 0.04 & 0.23 \\
\hline 33 & 4.8 & 5.5 & 23.11 & 0.05 & 22.84 & 0.05 & 0.26 \\
\hline 34 & 4.5 & -7.4 & 23.19 & 0.05 & 22.99 & 0.05 & 0.34 \\
\hline 35 & -0.4 & 4.7 & 23.83 & 0.07 & 22.91 & 0.04 & 0.03 \\
\hline 36 & -11.4 & 5.0 & 23.31 & 0.05 & 23.19 & 0.05 & 0.21 \\
\hline 37 & 16.5 & 9.9 & 23.68 & 0.05 & 23.38 & 0.04 & 0.15 \\
\hline 38 & 5.5 & 8.1 & 24.00 & 0.11 & 23.74 & 0.31 & $\ldots$ \\
\hline 39 & 12.3 & 5.5 & 23.71 & 0.06 & 23.57 & 0.06 & 0.16 \\
\hline 40 & -4.6 & -10.7 & 23.46 & 0.05 & 23.16 & 0.04 & 0.21 \\
\hline 41 & 19.1 & -6.7 & 23.61 & 0.06 & 23.41 & 0.06 & 0.25 \\
\hline 42 & 8.7 & -10.4 & 24.36 & 0.07 & 24.02 & 0.06 & $\ldots$ \\
\hline 43 & -1.5 & 15.4 & 24.32 & 0.07 & 24.13 & 0.07 & $\ldots$ \\
\hline 44 & 8.9 & 4.4 & 24.33 & 0.21 & 24.15 & 0.35 & $\cdots$ \\
\hline 45 & -11.8 & -14.6 & 24.38 & 0.06 & 24.08 & 0.06 & $\ldots$ \\
\hline 46 & -13.2 & -4.6 & 23.84 & 0.08 & 23.43 & 0.05 & 0.88 \\
\hline 47 & 8.8 & -12.1 & 23.84 & 0.07 & 23.64 & 0.06 & 0.24 \\
\hline 48 & 8.5 & -13.3 & 24.48 & 0.08 & 24.10 & 0.06 & $\cdots$ \\
\hline 49 & -10.0 & -5.7 & 24.60 & 0.09 & 24.28 & 0.09 & $\ldots$ \\
\hline 51 & -11.5 & 4.5 & 24.56 & 0.09 & 24.43 & 0.10 & $\cdots$ \\
\hline 52 & 2.6 & 7.6 & 24.56 & 0.09 & 23.98 & 0.07 & $\ldots$ \\
\hline 53 & 12.5 & 3.5 & 24.70 & 0.11 & 24.32 & 0.09 & $\cdots$ \\
\hline 54 & -5.1 & 7.1 & 24.67 & 0.14 & 24.25 & 0.09 & $\cdots$ \\
\hline 55 & -4.7 & -15.8 & 24.69 & 0.09 & 24.39 & 0.08 & $\ldots$ \\
\hline 56 & -11.1 & -4.9 & 24.70 & 0.09 & 24.44 & 0.09 & $\ldots$ \\
\hline 57 & 11.8 & 8.9 & 24.90 & 0.11 & 24.55 & 0.09 & $\ldots$ \\
\hline 58 & 13.0 & -0.3 & 24.15 & 0.10 & 22.97 & 0.04 & 0.49 \\
\hline 59 & -4.4 & 8.1 & 24.96 & 0.13 & 24.39 & 0.10 & $\ldots$ \\
\hline 60 & 1.0 & -16.2 & 24.98 & 0.14 & 24.57 & 0.12 & $\cdots$ \\
\hline 61 & -1.8 & -15.1 & 25.06 & 0.12 & 24.78 & 0.11 & $\ldots$ \\
\hline 62 & 13.1 & 3.8 & 25.08 & 0.17 & 24.68 & 0.15 & $\cdots$ \\
\hline 63 & 10.8 & -7.5 & 24.96 & 0.12 & 24.31 & 0.07 & $\ldots$ \\
\hline 64 & 5.0 & -13.2 & 25.05 & 0.12 & 24.51 & 0.09 & $\cdots$ \\
\hline 65 & 9.7 & 4.0 & 25.14 & 0.18 & 24.56 & 0.13 & $\ldots$ \\
\hline 66 & 5.3 & -7.4 & 25.46 & 0.33 & 25.01 & 0.30 & $\cdots$ \\
\hline 67 & 4.1 & -9.2 & 25.20 & 0.14 & 24.62 & 0.11 & $\ldots$ \\
\hline 68 & 9.9 & -10.4 & 25.19 & 0.13 & 24.92 & 0.13 & $\ldots$ \\
\hline 69 & 13.8 & -5.8 & 25.29 & 0.14 & 24.47 & 0.08 & $\cdots$ \\
\hline 70 & -8.0 & -4.2 & 25.68 & 0.29 & 23.80 & 0.05 & 0.10 \\
\hline 71 & 9.5 & -10.6 & 25.51 & 0.26 & 24.99 & 0.19 & $\cdots$ \\
\hline 72 & 1.0 & -15.5 & 25.68 & 0.24 & 25.02 & 0.14 & $\cdots$ \\
\hline
\end{tabular}


TABLE 5. NGC 6052 objects.

\begin{tabular}{|c|c|c|c|c|c|c|c|}
\hline ID & $\Delta \alpha$ & $\Delta \delta$ & V & $\sigma_{V}$ & $R$ & $\sigma_{R}$ & $m_{1}-m_{2}$ \\
\hline 1 & 5.4 & -2.8 & 18.86 & 0.00 & 18.91 & 0.00 & 0.21 \\
\hline 2 & -4.4 & -0.2 & 19.95 & 0.01 & 19.82 & 0.01 & 0.15 \\
\hline 3 & -0.6 & 1.0 & 21.00 & 0.07 & 20.77 & 0.09 & $\ldots$ \\
\hline 4 & -10.7 & 8.8 & 20.67 & 0.01 & 20.65 & 0.01 & 0.37 \\
\hline 5 & 5.2 & 13.4 & 20.65 & 0.01 & 20.73 & 0.01 & 0.41 \\
\hline 6 & 7.0 & -1.5 & 21.14 & 0.04 & 20.77 & 0.03 & 0.49 \\
\hline 7 & 6.5 & -0.1 & 21.20 & 0.05 & 21.07 & 0.05 & 0.34 \\
\hline 8 & 4.6 & -11.6 & 21.15 & 0.03 & 20.82 & 0.03 & 0.44 \\
\hline 9 & 5.5 & 1.2 & 21.34 & 0.02 & 20.98 & 0.02 & 0.56 \\
\hline 10 & -5.1 & -8.4 & 21.26 & 0.02 & 21.15 & 0.02 & 0.60 \\
\hline 11 & 3.2 & -7.8 & 21.80 & 0.03 & 21.54 & 0.03 & 0.15 \\
\hline 12 & -1.3 & -9.9 & 21.62 & 0.03 & 21.45 & 0.03 & 0.42 \\
\hline 13 & -2.3 & 3.2 & 21.58 & 0.02 & 20.89 & 0.01 & 0.69 \\
\hline 14 & 1.0 & 7.6 & 21.60 & 0.03 & 21.01 & 0.03 & 0.57 \\
\hline 15 & -4.6 & -13.1 & 21.67 & 0.02 & 21.69 & 0.02 & 0.39 \\
\hline 16 & 2.1 & 1.4 & 22.45 & 0.07 & 22.23 & 0.10 & $\ldots$ \\
\hline 17 & 5.7 & -0.4 & 22.68 & 0.16 & 22.25 & 0.13 & $\cdots$ \\
\hline 18 & -0.6 & -1.0 & 22.54 & 0.22 & 22.27 & 0.26 & $\cdots$ \\
\hline 20 & 4.8 & -1.1 & 22.56 & 0.07 & 22.31 & 0.07 & $\ldots$ \\
\hline 21 & 10.5 & -10.4 & 21.95 & 0.03 & 21.84 & 0.03 & 0.46 \\
\hline 22 & -2.8 & -8.4 & 22.05 & 0.02 & 21.80 & 0.02 & 0.44 \\
\hline 23 & -2.9 & -0.2 & 22.33 & 0.06 & 22.11 & 0.06 & 0.16 \\
\hline 24 & 2.2 & 14.8 & 22.05 & 0.04 & 21.78 & 0.03 & 0.44 \\
\hline 25 & -5.3 & -0.4 & 22.91 & 0.35 & 22.78 & 0.49 & $\ldots$ \\
\hline 26 & -8.1 & 9.7 & 22.12 & 0.02 & 21.76 & 0.02 & 0.52 \\
\hline 27 & 1.8 & 8.4 & 22.04 & 0.06 & 21.25 & 0.03 & 0.41 \\
\hline 28 & -4.6 & -3.5 & 23.06 & 0.09 & 22.60 & 0.08 & $\ldots$ \\
\hline 29 & 2.0 & 18.0 & 22.57 & 0.03 & 22.30 & 0.02 & 0.17 \\
\hline 30 & 5.4 & -8.7 & 22.16 & 0.06 & 21.70 & 0.05 & 0.28 \\
\hline 31 & 5.6 & -8.9 & 22.80 & 0.07 & 22.24 & 0.06 & $\ldots$ \\
\hline 33 & 4.4 & -7.5 & 23.10 & 0.06 & 22.84 & 0.07 & $\cdots$ \\
\hline 34 & 6.4 & 0.1 & 22.77 & 0.11 & 22.11 & 0.08 & $\ldots$ \\
\hline 35 & 1.7 & 16.2 & 23.18 & 0.06 & 22.85 & 0.07 & $\cdots$ \\
\hline 36 & -5.1 & 1.9 & 22.72 & 0.06 & 22.08 & 0.03 & 0.57 \\
\hline 37 & 1.1 & -1.6 & 23.72 & 0.17 & 23.43 & 0.22 & $\ldots$ \\
\hline 38 & 9.5 & -9.7 & 22.68 & 0.04 & 22.28 & 0.03 & 0.46 \\
\hline 39 & 6.3 & 7.0 & 23.44 & 0.10 & 23.02 & 0.10 & $\ldots$ \\
\hline 40 & 7.5 & -13.3 & 22.78 & 0.05 & 22.61 & 0.06 & 0.34 \\
\hline 41 & -3.0 & 0.1 & 23.17 & 0.12 & 22.69 & 0.10 & $\cdots$ \\
\hline 42 & -1.1 & -9.1 & 23.48 & 0.15 & 23.00 & 0.15 & $\ldots$ \\
\hline 43 & -11.2 & -3.4 & 23.25 & 0.04 & 22.95 & 0.03 & 0.15 \\
\hline 44 & 4.5 & -8.9 & 23.72 & 0.17 & 23.04 & 0.15 & $\cdots$ \\
\hline 45 & 5.7 & 8.3 & 23.47 & 0.07 & 23.18 & 0.08 & $\ldots$ \\
\hline 46 & 0.2 & -12.6 & 23.38 & 0.07 & 23.09 & 0.06 & 0.13 \\
\hline 47 & 8.4 & -13.8 & 23.45 & 0.09 & 22.94 & 0.07 & $\ldots$ \\
\hline 48 & 8.6 & -13.0 & 23.39 & 0.08 & 23.01 & 0.09 & $\cdots$ \\
\hline 49 & 0.0 & -11.7 & 23.63 & 0.07 & 23.28 & 0.08 & $\ldots$ \\
\hline 50 & 3.3 & 8.5 & 23.97 & 0.16 & 23.98 & 0.28 & $\cdots$ \\
\hline 51 & 2.1 & -2.3 & 23.65 & 0.12 & 23.38 & 0.14 & $\ldots$ \\
\hline 52 & 2.4 & -14.1 & 23.37 & 0.05 & 23.22 & 0.05 & 0.18 \\
\hline 53 & 0.4 & -7.1 & 23.87 & 0.08 & 23.65 & 0.08 & $\cdots$ \\
\hline 54 & 8.6 & -13.2 & 23.08 & 0.07 & 22.68 & 0.06 & 0.23 \\
\hline 55 & 1.1 & -2.7 & 23.79 & 0.12 & 23.55 & 0.15 & $\ldots$ \\
\hline 56 & 3.5 & 15.6 & 24.07 & 0.08 & 23.54 & 0.08 & $\ldots$ \\
\hline 57 & -2.9 & -15.8 & 23.85 & 0.08 & 23.41 & 0.06 & 0.10 \\
\hline 58 & 9.1 & -12.3 & 23.88 & 0.13 & 23.25 & 0.10 & $\ldots$ \\
\hline 59 & 0.1 & 16.7 & 24.28 & 0.09 & 23.91 & 0.07 & $\ldots$ \\
\hline 60 & 10.2 & 11.0 & 24.11 & 0.06 & 23.90 & 0.06 & 0.11 \\
\hline 61 & 7.8 & 8.8 & 24.24 & 0.07 & 23.76 & 0.06 & $\cdots$ \\
\hline 62 & 11.7 & 2.1 & 23.84 & 0.06 & 23.29 & 0.04 & 0.18 \\
\hline 63 & -0.5 & -7.2 & 24.32 & 0.14 & 24.07 & 0.14 & $\cdots$ \\
\hline 64 & -3.7 & -10.7 & 24.42 & 0.08 & 24.18 & 0.07 & $\cdots$ \\
\hline 65 & 2.8 & -7.0 & 24.60 & 0.26 & 23.80 & 0.20 & $\ldots$ \\
\hline 66 & 12.7 & -6.4 & 24.43 & 0.07 & 24.06 & 0.06 & $\ldots$ \\
\hline 67 & 1.4 & -11.9 & 24.62 & 0.13 & 24.25 & 0.11 & $\ldots$ \\
\hline 68 & 7.8 & 14.0 & 24.69 & 0.09 & 24.33 & 0.08 & $\cdots$ \\
\hline 69 & 0.8 & -7.0 & 24.56 & 0.11 & 24.21 & 0.09 & $\ldots$ \\
\hline 70 & 8.6 & 10.7 & 24.86 & 0.10 & 24.32 & 0.07 & $\ldots$ \\
\hline 71 & 9.4 & 9.5 & 24.69 & 0.09 & 24.21 & 0.07 & $\ldots$ \\
\hline 72 & -1.4 & 16.8 & 25.04 & 0.14 & 24.54 & 0.11 & $\ldots$ \\
\hline 73 & 10.8 & -7.2 & 25.14 & 0.14 & 24.58 & 0.12 & $\ldots$ \\
\hline
\end{tabular}


TABLE 6. Abell 496 objects.

\begin{tabular}{|c|c|c|c|c|c|c|c|}
\hline ID & $\Delta \alpha$ & $\Delta \delta$ & $V$ & $\sigma_{V}$ & $R$ & $\sigma_{R}$ & $m_{1}-m_{2}$ \\
\hline 1 & 2.0 & -5.0 & 23.62 & 0.05 & 23.05 & 0.05 & 0.17 \\
\hline 2 & 11.1 & -16.7 & 24.11 & 0.05 & 23.54 & 0.04 & 0.15 \\
\hline 3 & 15.5 & -8.6 & 24.01 & 0.05 & 23.56 & 0.04 & 0.19 \\
\hline 4 & -8.1 & -12.4 & 24.57 & 0.05 & 24.33 & 0.06 & 0.02 \\
\hline 5 & 3.1 & 10.7 & 24.61 & 0.07 & 24.05 & 0.05 & 0.11 \\
\hline 6 & 10.3 & -1.1 & 24.70 & 0.12 & 23.98 & 0.06 & 0.04 \\
\hline 7 & -8.1 & -6.0 & 24.52 & 0.08 & 23.92 & 0.05 & 0.17 \\
\hline 8 & 6.5 & -12.1 & 24.87 & 0.08 & 24.29 & 0.05 & 0.10 \\
\hline 9 & -1.8 & -5.9 & 24.92 & 0.10 & 24.24 & 0.09 & $\ldots$ \\
\hline 10 & -10.9 & 6.2 & 24.98 & 0.09 & 24.43 & 0.06 & 0.09 \\
\hline 11 & 15.3 & 5.7 & 25.13 & 0.12 & 24.43 & 0.07 & $\cdots$ \\
\hline 12 & -6.1 & -16.7 & 25.20 & 0.09 & 24.68 & 0.07 & $\ldots$ \\
\hline 13 & -10.2 & -3.1 & 25.15 & 0.11 & 24.37 & 0.06 & 0.12 \\
\hline 14 & -10.3 & -6.9 & 25.33 & 0.10 & 24.65 & 0.07 & $\ldots$ \\
\hline 15 & 2.6 & -7.3 & 25.27 & 0.12 & 24.52 & 0.09 & $\ldots$ \\
\hline 16 & 9.0 & -4.0 & 25.40 & 0.12 & 24.75 & 0.08 & $\cdots$ \\
\hline 17 & 3.2 & -9.2 & 25.49 & 0.14 & 24.94 & 0.11 & $\cdots$ \\
\hline 18 & -15.5 & -7.1 & 25.46 & 0.12 & 25.05 & 0.08 & $\cdots$ \\
\hline 19 & -2.8 & 4.2 & 25.80 & 0.28 & 24.95 & 0.21 & $\cdots$ \\
\hline 20 & 4.6 & 12.2 & 25.55 & 0.13 & 24.93 & 0.09 & $\ldots$ \\
\hline 21 & 0.4 & 6.1 & 25.52 & 0.18 & 24.68 & 0.13 & $\cdots$ \\
\hline 22 & 4.9 & -17.8 & 25.58 & 0.13 & 25.03 & 0.09 & $\cdots$ \\
\hline 23 & 13.9 & 4.1 & 25.64 & 0.14 & 24.95 & 0.09 & $\ldots$ \\
\hline 24 & 10.9 & -3.7 & 25.66 & 0.14 & 25.19 & 0.12 & $\cdots$ \\
\hline 25 & 1.9 & -13.5 & 25.63 & 0.14 & 24.95 & 0.09 & $\cdots$ \\
\hline 27 & 12.8 & -3.3 & 25.74 & 0.15 & 25.29 & 0.12 & $\cdots$ \\
\hline 28 & 0.8 & -4.4 & 25.77 & 0.31 & 24.99 & 0.28 & $\cdots$ \\
\hline 30 & -1.5 & 14.1 & 26.00 & 0.19 & 25.46 & 0.16 & $\ldots$ \\
\hline 31 & 11.4 & -14.7 & 25.95 & 0.22 & 25.25 & 0.16 & $\ldots$ \\
\hline 32 & 15.4 & -8.1 & 25.82 & 0.15 & 25.00 & 0.08 & $\ldots$ \\
\hline 33 & -14.9 & -5.0 & 26.08 & 0.19 & 25.26 & 0.10 & $\ldots$ \\
\hline 34 & 1.1 & 13.3 & 26.24 & 0.24 & 25.55 & 0.15 & $\cdots$ \\
\hline 35 & 7.8 & -1.3 & 26.17 & 0.26 & 25.29 & 0.15 & $\cdots$ \\
\hline 36 & -2.7 & 15.6 & 26.34 & 0.27 & 25.49 & 0.13 & $\ldots$ \\
\hline 37 & 7.8 & -1.0 & 26.23 & 0.27 & 25.54 & 0.21 & $\ldots$ \\
\hline 38 & 0.3 & 16.6 & 26.71 & 0.43 & 25.60 & 0.13 & $\cdots$ \\
\hline 39 & -6.4 & 13.0 & 25.91 & 0.19 & 25.07 & 0.11 & $\cdots$ \\
\hline
\end{tabular}

sizes not change significantly over 10 Gyr. Survival issues are difficult to address without a knowledge of the orbits of the clusters and their IMFs.

Since the current observations are not especially deep, we have not attempted to construct a luminosity function for the observed clusters. Consequently, the question of whether the luminosity function of the cluster system in NGC 3597 resembles that of the Galactic globular cluster system remains open. As individuals, however, the NGC 3597 clusters are probably comparable to globulars.

The brightest object at $V \sim 20$ is red and unresolved, and it is most likely a foreground star. The fainter red objects may be resolved older clusters or they may be young clusters that have significant internal reddening.

\section{$3.4 N G C 6052$}

NGC 6052 also shows a population of bright blue objects. Some of these are brighter and bluer than those seen in NGC 3597. However, most of these objects are quite extended; indeed, the observed angular sizes are almost all larger than those in NGC 3597, even though NGC 6052 is at a larger distance. Many of the $m_{1}-m_{2}$ values require values of $r_{0}$ on the order of 10 parsecs.
Consequently, although these objects appear to have colors and brightnesses suggesting that they are young massive clusters, most of them do not appear to be as compact as Galactic globulars. In addition, most of these objects are not isolated from the background galaxy; many are not symmetrical and their appearance suggests that these are in active regions of star formation. Their blue colors suggest young ages, and this is supported by the observation that most of the objects are located in regions where there is $\mathrm{H} \alpha$ emission (Pogge, private communication). We suspect that these are massive associations with associated $\mathrm{H}$ II regions.

There are a few objects which do appear to be as compact as globulars; of particular interest are the two brightest objects. If we adopt an age of 5 million years from the observed colors, the masses of the two brightest clusters would be $\sim 1.6 \times 10^{6}$ and $\sim 6 \times 10^{5} M_{\odot}$. It is possible that some of the other clusters may also have massive compact cores; we cannot tell from these data because most of the objects are embedded in diffuse emission.

\subsection{Abell 496}

We see no evidence for young massive compact clusters in this cooling flow elliptical; there is no population of bright 
TABLE 7. Abell 1795.

\begin{tabular}{|c|c|c|c|c|c|c|c|}
\hline ID & $\Delta \alpha$ & $\Delta \delta$ & $V$ & $\sigma_{V}$ & $R$ & $\sigma_{R}$ & $m_{1}-m_{2}$ \\
\hline 2 & -0.6 & 1.4 & 23.43 & 0.11 & 23.28 & 0.21 & $\ldots$ \\
\hline 3 & 16.6 & 0.7 & 23.88 & 0.03 & 23.34 & 0.02 & $\ldots$ \\
\hline 4 & -9.6 & -3.4 & 23.79 & 0.04 & 23.18 & 0.03 & 0.17 \\
\hline 5 & -1.5 & 2.7 & 24.72 & 0.16 & 24.60 & 0.28 & $\ldots$ \\
\hline 6 & -2.5 & 0.6 & 24.95 & 0.19 & 24.96 & 0.48 & $\ldots$ \\
\hline 7 & 10.0 & -2.8 & 24.94 & 0.07 & 24.02 & 0.03 & 0.03 \\
\hline 8 & 0.2 & 15.7 & 25.24 & 0.12 & 24.58 & 0.10 & $\cdots$ \\
\hline 9 & -2.0 & 2.5 & 24.80 & 0.15 & 24.50 & 0.23 & $\cdots$ \\
\hline 10 & -3.2 & 3.9 & 25.07 & 0.39 & 24.84 & 0.39 & $\cdots$ \\
\hline 12 & 0.7 & 5.0 & 25.65 & 0.17 & 25.38 & 0.24 & $\cdots$ \\
\hline 13 & -2.7 & -1.0 & 25.38 & 0.26 & 25.19 & 0.49 & $\cdots$ \\
\hline 15 & 4.3 & 7.8 & 25.96 & 0.17 & 25.30 & 0.11 & $\ldots$ \\
\hline 16 & -10.1 & 7.2 & 25.68 & 0.13 & 25.56 & 0.14 & $\cdots$ \\
\hline 17 & -9.8 & -1.1 & 25.73 & 0.14 & 25.14 & 0.09 & $\cdots$ \\
\hline 18 & 4.7 & 4.7 & 25.74 & 0.15 & 25.26 & 0.13 & $\cdots$ \\
\hline 19 & -5.1 & -0.2 & 26.03 & 0.23 & 25.24 & 0.17 & $\cdots$ \\
\hline 20 & -0.3 & 17.9 & 25.80 & 0.14 & 25.25 & 0.10 & $\cdots$ \\
\hline 21 & 5.1 & 11.5 & 26.19 & 0.20 & 25.55 & 0.15 & $\cdots$ \\
\hline 22 & -16.7 & 1.7 & 26.09 & 0.19 & 25.29 & 0.09 & $\cdots$ \\
\hline 23 & -13.4 & 8.6 & 26.46 & 0.26 & 25.53 & 0.11 & $\cdots$ \\
\hline 24 & 3.5 & 9.4 & 26.14 & 0.19 & 25.52 & 0.14 & $\cdots$ \\
\hline 25 & 9.8 & 6.6 & 26.30 & 0.23 & 25.73 & 0.16 & $\cdots$ \\
\hline 26 & 12.4 & 0.5 & 26.49 & 0.26 & 25.56 & 0.12 & $\cdots$ \\
\hline 27 & -9.9 & 0.5 & 26.50 & 0.27 & 25.72 & 0.15 & $\cdots$ \\
\hline 28 & 9.9 & 2.9 & 26.59 & 0.29 & 25.68 & 0.14 & $\cdots$ \\
\hline 29 & -9.0 & 6.7 & 26.62 & 0.32 & 25.49 & 0.11 & $\cdots$ \\
\hline 30 & -10.2 & 7.6 & 27.01 & 0.48 & 26.06 & 0.20 & $\ldots$ \\
\hline
\end{tabular}

blue objects. There is a population of red objects ranging up to $M_{V} \sim-12$. These are likely to be old globular clusters around the galaxy; if this galaxy has a specific frequency similar to M87, such clusters are expected. Figure 6 suggests that the objects in Abell 496 may be marginally resolved. This is plausible as most of the inferred core radii are less than 0.1 pixels, or $3 \mathrm{pc}$. The one more resolved object with a slightly bluer color may be a background galaxy. There may be one unresolved blue source, but even if this is a young cluster associated with Abell 496, the maximum mass is $\sim 3 \times 10^{5} M_{\odot}$ for a maximum age of $10^{8}$ years.

\subsection{Abell 1795}

The central galaxy in Abell 1795 shows some remarkable structure, with a large dust lane near the center of the galaxy and several blue filamentary structures. These are discussed in more detail in Pinkney et al. (1996). As in NGC 1275, it is possible that these features are a result of a fairly recent merger. There are several "knots" around the dust lane which may be clusters of stars. Accurate measurements of these are difficult because of unknown background levels and extinction.

Perhaps the most interesting source is the blue source at $M_{V} \sim-13.6$. This object is near the center of the galaxy and accurate photometry is difficult because of the bright background. This is a candidate young cluster. For a color of $V-R \sim 0.1$, the inferred age is $2 \times 10^{7}$ years and the inferred mass is $\sim 1.2 \times 10^{6} M_{\odot}$. The fainter bluer objects are all found near the filamentary structure and the dust lane; it is possible that they are young star forming regions. If these objects are clusters, their masses could be comparable to Galactic globulars. At the distance of Abell 1795, it is impossible to determine whether any of these objects are compact enough to be comparable to globular clusters but we

TABLE 8. Abell 2029 objects.

\begin{tabular}{rrrrrrrr}
\hline \hline ID & \multicolumn{1}{c}{$\Delta \alpha$} & \multicolumn{1}{c}{$\Delta \delta$} & $V$ & $\sigma_{V}$ & $R$ & $\sigma_{R}$ & $m_{1}-m_{2}$ \\
\hline 3 & -2.6 & -3.8 & 24.94 & 0.18 & 24.22 & 0.11 & $\ldots$ \\
4 & -5.3 & 7.5 & 24.99 & 0.14 & 24.34 & 0.05 & 0.09 \\
5 & -8.9 & -3.0 & 25.56 & 0.16 & 25.19 & 0.11 & $\cdots$ \\
7 & -3.7 & -6.9 & 25.73 & 0.21 & 25.32 & 0.15 & $\cdots$ \\
8 & -4.6 & 2.3 & 25.36 & 0.29 & 24.54 & 0.10 & $\cdots$ \\
9 & 3.5 & 5.1 & 25.64 & 0.29 & 24.96 & 0.14 & $\ldots$ \\
10 & 3.9 & -12.7 & 25.33 & 0.23 & 24.69 & 0.06 & 0.12 \\
11 & 11.4 & 19.1 & 25.75 & 0.31 & 25.14 & 0.08 & $\cdots$ \\
12 & 3.7 & 7.6 & 26.07 & 0.43 & 25.33 & 0.13 & $\cdots$ \\
15 & -11.3 & -5.4 & 26.30 & 0.50 & 25.51 & 0.10 & $\cdots$ \\
17 & 8.7 & 9.1 & 26.32 & 0.44 & 25.69 & 0.14 & $\cdots$ \\
\hline
\end{tabular}


TABLE 9. Abell 2597 objects.

\begin{tabular}{rrrrrrrr}
\hline \hline ID & \multicolumn{1}{c}{$\Delta \alpha$} & $\Delta \delta$ & $V$ & $\sigma_{V}$ & $R$ & $\sigma_{R}$ & $m_{1}-m_{2}$ \\
\hline 1 & -5.6 & -3.8 & 23.55 & 0.06 & 23.03 & 0.02 & 0.20 \\
2 & -8.2 & -8.7 & 24.10 & 0.11 & 23.09 & 0.01 & 0.00 \\
4 & 7.1 & 0.3 & 25.06 & 0.15 & 24.50 & 0.05 & 0.10 \\
5 & 11.1 & -8.3 & 25.32 & 0.18 & 24.69 & 0.05 & 0.02 \\
6 & -4.8 & 6.7 & 25.42 & 0.27 & 25.01 & 0.08 & $\ldots$ \\
7 & -2.0 & -10.6 & 25.65 & 0.21 & 25.11 & 0.08 & $\ldots$ \\
8 & -13.6 & -1.6 & 25.77 & 0.34 & 25.44 & 0.11 & $\ldots$ \\
10 & -0.6 & -4.7 & 25.76 & 0.28 & 25.14 & 0.12 & $\ldots$ \\
11 & 12.5 & -8.5 & 25.91 & 0.26 & 25.42 & 0.09 & $\cdots$ \\
13 & 12.6 & -7.2 & 26.11 & 0.37 & 25.47 & 0.09 & $\ldots$ \\
14 & 9.4 & 0.5 & 26.01 & 0.22 & 25.72 & 0.12 & $\ldots$ \\
\hline \hline
\end{tabular}

cannot exclude this possibility. From the current observations, it is unclear whether these blue objects are clusters that recently formed in the central galaxy environment (i.e., related to the cooling flow), whether they are part of another galaxy seen in the process of merging with the central galaxy, or whether they are clusters that recently formed as a result of a possible merger. In many ways, Abell 1795 is similar to NGC 1275 , and the peculiarities of both make it hard to determine the origin of their massive clusters.

There are two red objects at $M_{V} \sim-13$. One of them appears to be resolved, and we suspect this may be a dwarf galaxy in the cluster or else a background galaxy. The other object appears unresolved and is probably either a fore-

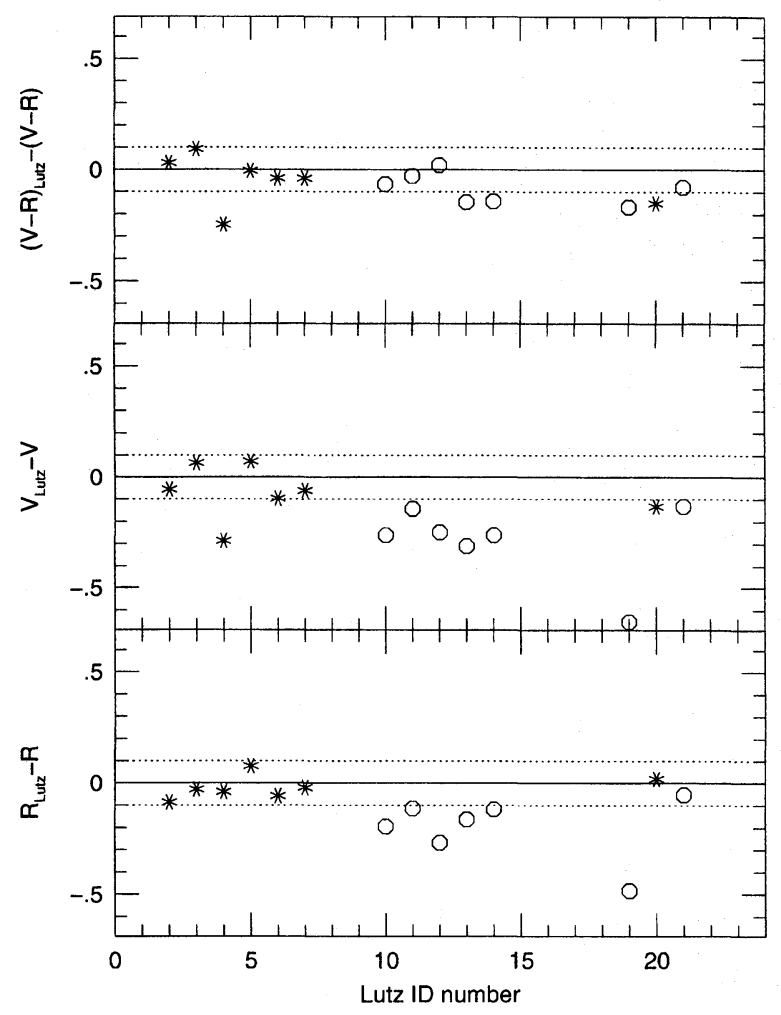

FIG. 7. Measured magnitudes and colors for objects in NGC 3597 compared with values obtained by Lutz (1991). Asterisks represent objects which are likely to be stars. ground star or a very bright old globular. Most of the fainter red objects are probably old globulars around Abell 1795.

\subsection{Abell 2029}

In the central galaxy in Abell 2029, all the objects seen are fairly red, with $V-R \sim 0.6$. These may be old globular clusters in Abell 2029, although they are rather bright. If these are old globulars, a large population of clusters probably exists in Abell 2029 in order to populate this brightness range. The fainter objects that appear blue have poorly determined colors with estimated errors of several tenths of a magnitude. There is no strong evidence for any young massive clusters.

\subsection{Abell 2597}

Abell 2597 shows two moderately bright objects, one of which appears partially resolved, and one which is unresolved. Neither is especially blue. The resolved object is another candidate dwarf or background galaxy and the unresolved object is likely to be a foreground star because of its color. Apart from these, the observed objects are probably all consistent with being old globular clusters in the system or foreground stars.

\section{CONCLUSIONS}

We have observed several interacting galaxies and several central galaxies in cooling flow clusters in an effort to find young massive star clusters preferentially in one of these environments. We find evidence for massive young clusters in both interacting galaxies in our sample, NGC 3597 and NGC 6052. In both galaxies, the star clusters are resolved by HST. The inferred sizes of most of the clusters in NGC 3597 and the two brightest clusters in NGC 6052 are probably comparable to globular clusters. The majority of the clusters in NGC 6052, however, appear more extended. The results for NGC 3597 confirm the suggestion of Lutz (1991) that the objects in this galaxy appear to be analogs of Galactic globulars, but seen at a younger age.

Most of the cooling flow clusters do not show evidence for compact young star clusters. The central galaxy in Abell 1795, however, is an exception, as it shows evidence for at least one massive young cluster, and possibly several more clusters of somewhat lower mass. Interestingly, Abell 1795 


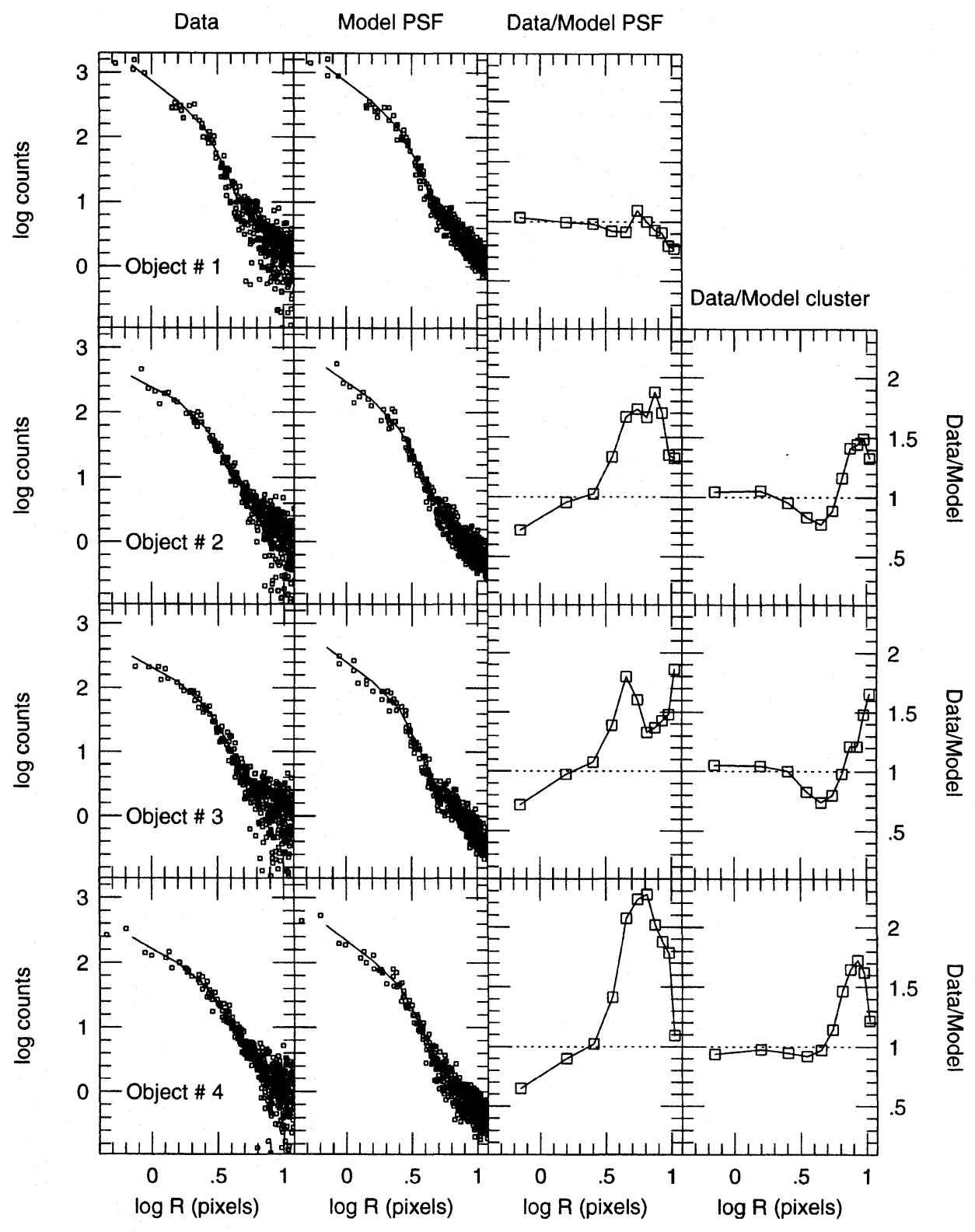

FIG. 8. Observed and model profiles for the four brightest objects in NGC 3597. The left panel shows the data with an azimuthally averaged profile. The second panel from left shows a model PSF at the same field location and pixel centering. The next panel shows the ratio of the observed profile to the model PSF. The right panel shows the ratio of the observed profile to a model cluster with $r_{0}=0.05$ pixels convolved with a model PSF.

shows the most evidence of star formation activity, with very blue central colors and the presence of dust (Pinkney et al. 1996; McNamara \& O'Connell 1993). Because of the structure seen in Abell 1795, it is difficult to know whether the presence of young clusters is associated with the cooling flow. Since the other cooling flow galaxies do not have any young clusters, it makes such an association less likely. A similar conclusion is drawn by Harris et al. (1995), who do not find any correlation between the number of clusters or the specific frequency and the x-ray properties of three additional central cluster galaxies.

These results continue to increase the number of galaxies in which young, compact, massive star clusters are found. These sites of massive cluster formation include interacting galaxies, dwarf galaxies, starburst galaxies, and ring galaxies (references in the Introduction). Generally, all sites of cluster formation appear to be sites of distributed star formation. It appears possible that massive cluster formation occurs wherever the general star formation rate is sufficiently high. The most extensive systems of young massive clusters appear to be occurring in interacting galaxies: most of the other galaxies show evidence for only a handful of clusters.

We are planning deeper observations to measure the cluster luminosity function in NGC 3597 to address the issue of 
the relative amount of star formation in clusters to star formation in unbound or loosely bound entities. This is especially relevant to the issue of whether the increased specific frequency of globulars in ellipticals is related to the formation of new cluster systems in interacting systems like NGC 3597 , since an increase in specific frequency requires star formation to occur preferentially in clusters (Harris et al. 1995). To address this issue properly requires an understanding of the faint end of the young cluster luminosity function, the probability of cluster survival, and a good estimate of the star formation rate outside the clusters.

The question of whether these objects are precursors to globular clusters also remains open, as the answer depends on how a globular cluster is defined. For example, do young globular clusters have to be in systems which have luminos- ity functions similar to Galactic globular clusters (van den Bergh 1995)? It is possible that the recently discovered clusters may individually be good analogs of individual globular clusters, but the systems of clusters may not necessarily be analogs of the old globular cluster systems. However, present day system properties of young compact massive clusters, or even properties of individual clusters, might differ from those of the Galactic globulars for a number of reasons (e.g., evolution, different chemical composition, different formation environments) even if the general formation mechanism was the same.

This work was supported in part by NASA under contract NAS7-918 to JPL.

\section{REFERENCES}

Bahcall, J. N., \& Soneira, R. M. 1981, ApJS, 47, 357

Barth, A. J., Ho, L. C., Filippenko, A. V., \& Sargent, W. L. W. 1995, AJ 110, 1009

Burstein, D., \& Heiles, C. 1984, APJS, 54, 33

Charlot, S., \& Bruzual, A. G. 1991, ApJ, 327, 126

Conti, P. S., \& and Vacca, W. D. 1994, ApJ, 423, L97

Couture, J., Harris, W. E., \& Allwright, J. W. B. 1990, ApJS, 73, 671

Crabtree, D. R., \& Smecker-Hane, T. A. 1994, BAAS 185, \#107.74

Djorgovski, G. 1993, ASP Conf. Ser. 50, 373

Fabian, A. C. 1992, ARA\&A, 32, 277

Harris, W. E., \& Racine, R. 1979, ARA\&A, 17, 241

Harris, W. E. Pritchet, C. J., \& McClure, R. D. 1995, ApJ, 441, 120

Hequet, J., Coupinot, G., \& Maucherat, A. J. 1987, A\&A, 183, 13

Holtzman, J. A., et al. 1995a, PASP, 107, 156

Holtzman, J. A., Burrows, C. J., Casertano, S., Hester, J. J., Trauger, J. T.,

Watson, A. M., \& Worthy, G. 1995b, PASP, 107, 1065

Holtzman, J. A., et al. 1992, AJ, 103, 691

Hunter, D. A., O'Connell, R. W., \& Gallagher, J. S. 1994, AJ, 108, 84

Lutz, D. 1991, A\&A, 245, 31

McNamara, B., \& O’Connell, R. W. 1993, AJ, 105, 417
McNamara, B., \& O'Connell, R. W. 1992, ApJ, 393, 579

Pinkney, J., Holtzman, J. A., Garasi, C., \& the WFPC2 team, 1996, ApJL (in press)

O'Connell, R. W., Gallagher, J. S., \& Hunter, D. A. 1994, ApJ, 433, 65

O'Connell, R. W., Gallagher, J. S., Hunter, D. A., \& Colley W. N. 1995, ApJ, 446, L10

Pryor, C., \& Meylan, G. 1993, ASP Conf. Ser., 50, 537

Reed, B. C., Hesser, J. E., \& Shawl, S. J. 1988, PASP, 100, 545

Richer, H. B., Crabtree, D. R., Fabian, A. C., \& Lin, D. N. C. 1993, AJ, 105, 877

Strom, S. E., Forte, J. C., Harris, W. E., Strom, K. M., Wells, D. C., \& Smith, M. G. 1981, ApJ, 245, 416

van den Bergh, S., Morbey, C., \& Pazder, J. 1991, ApJ, 375, 594

van den Bergh, S. 1995, ApJ, 450, 27

Watson, A. M., Gallagher, J. S., Holtzman, J. A., Hester, J. J., \& the WFPC2 IDT, 1996, AJ, 112, 534

Whitmore, B., \& Schweizer, F. 1995, AJ, 109, 960

Whitmore, B. C., Schweizer, F., Leitherer, C., Borne, K., \& Rober, C. 1993, AJ, 106, 1354

Zepf, K., \& Ashman, K. 1993, MNRAS, 165, 611 


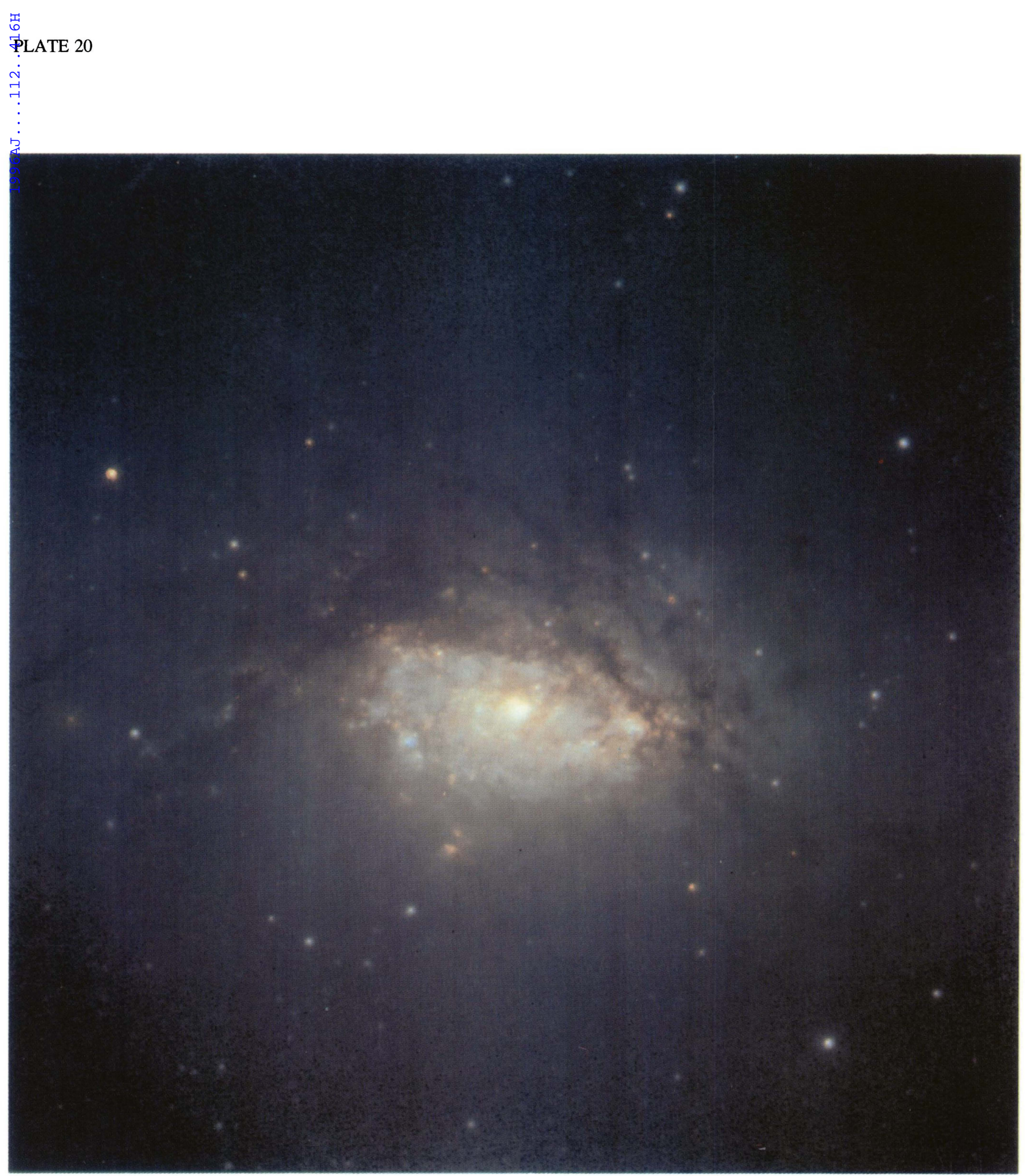

FIG. 1. A color image of NGC 3597 made from the WFPC2 F555W and F702W images. North is up and east is to the left.

Holtzman et al. (see page 418) 


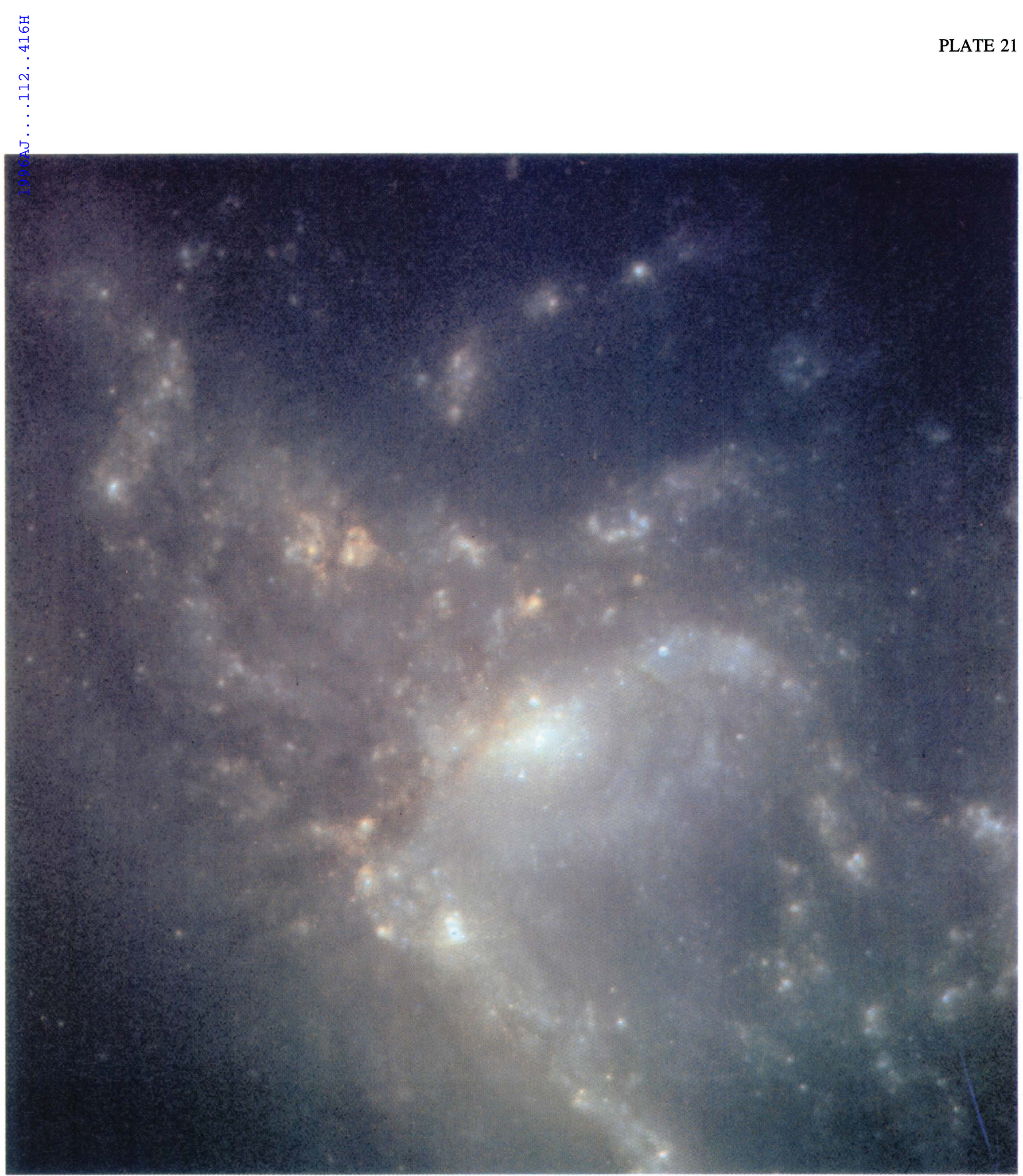

FIG. 2. A color image of NGC 6052 made from the WFPC2 F555W and F702W images. North is located 38 degrees counter-clockwise from vertical.

Holtzman et al. (see page 418) 